\title{
AN GROUP BEHAVIOR MOBILITY MODEL FOR OPPORTUNISTIC NETWORKS
}

\author{
GuoDong KANG ${ }^{1}$ and GuoLiang KANG ${ }^{2}$ \\ ${ }^{1}$ DFH Satellite Co., Ltd., 100094, Beijing, China \\ kongton584@163.com \\ ${ }^{2}$ University of Technology, Sydney 15 Broadway, Ultimo NSW 2007 \\ kgl.prml@gmail.com
}

\begin{abstract}
Mobility is regarded as a network transport mechanism for distributing data in many networks. However, many mobility models ignore the fact that peer nodes often carried by people and thus move in group pattern according to some kind of social relation. In this paper, we propose one mobility model based on group behavior character which derives from real movement scenario in daily life. This paper also gives the character analysis of this mobility model and compares with the classic Random Waypoint Mobility model.
\end{abstract}

\section{KEYWORDS}

Mobility model, Group behavior, Opportunistic network

\section{INTRODUCTION}

Nowadays mobility is playing an important role more and more in many wireless networks such as mobile ad-hoc networking (MANET) and delay-tolerant networking (DTN) [2, 3]. One common important fact for these opportunistic networks is that mobile devices among them are often carried by people. From this point of view, the mobility of mobile devices is exactly the same as the movement of persons who are taking them. The idea of mobility structure in this paper comes from people's movement custom or character in everyday life.

Usually we can observe one familiar scenario: persons in the same group often go to some place together then to another place together meantime everyone has his own walking or moving style. How to construct this kind of mobility is the main work of this paper.

To construct this kind of mobility model, we have to face two questions: how to allocate the given nodes into different groups according to certain method; how to describe and construct nodes' common group mobility behavior and every node's individual mobility behavior. The mobility model proposed in this paper thoroughly considers nodes' individual realistic movement character combing common group character based group division method.

David C. Wyld et al. (Eds) : WiMo, ITCSE, ICAIT, NC - 2015

pp. 81-89, 2015. @ CS \& IT-CSCP 2015

DOI : $10.5121 / \mathrm{csit} .2015 .51008$ 
The rest of this paper is organized as follows: Section II describes the allocation method of mobile nodes in opportunistic network. Section III proposes the setup method of the mobility models based on common group-behavior character and individual self-behavior character. Section IV gives analysis of the proposed new mobility model. Conclusion is given in Section V.

\section{GROUP ALLOCATION METHOD}

The group allocation method can be undertaken as following two steps: Firstly, the network should find and construct the relations among whole nodes in the form of mathematic matrix which will be regarded as the basis of second step. Then whole nodes are allocated into different groups according to the relations founded in first step.

\subsection{Node Relation Setup}

Normally, there is some kind of social or biological relations among those nodes since they are carried by people. To set up this kind of mutual relation, we adopt the classical method of representing social or biological network, weighted graphs. The strength of mutual relation between any node pair is represented using a value in the range $[0,1]$. As a consequence, the network internal relation can be described as a relation matrix with a dimension of $\mathrm{N} * \mathrm{~N}$ where $\mathrm{N}$ is the total number of nodes in the network. At present, there are several models that describe the key properties of real-life network, such as random graph, small world, etc. Some research work show that the properties of these random graphs, such as path lengths, clustering coefficients, can't be regarded as accurate models of realistic networks $[5,6,7]$. Here, we choose the geometric random graph to be the network model. In this kind of model, the geometry relations of nodes have strong association with the social relation of nodes. That means when any two nodes are in the radio range of each other, the social relation exists. On the contrary, since they even can't communicate with each other, we think that there is no any social interaction between them. So when the Euclidean distance between any two nodes is smaller than radio range R, the corresponding element of the social relation matrix $\mathrm{M}$ is set to be 1 or else set to be 0 as follows.

$$
m_{i, j}=\left\{\begin{array}{l}
1 \text { if } i \neq j \text { and }\left\|P_{i}-P_{j}\right\| \leq R, \\
0 \text { otherwise }
\end{array}\right.
$$

It should be emphasized that the relations value of one node and itself is regarded to be zero in the matrix. In [6] it is shown that in two or three dimensional area using Euclidean norm can supply surprisingly accurate reproductions of many features of real biological networks.

For example, 100 nodes are uniformly distributed as shown figure 1. Lines are used to represent the mutual relations among these nodes whose radio range is assumed to be 10 meters here.

\subsection{Nodes Group Allocation}

Once the relation matrix $\mathrm{M}$ is obtained, groups can be detected. Group structure is one of the common characters in many real networks. However, finding group structures within an arbitrary network is an acknowledged difficult task. A lot of work has been done on that. Currently, there are several methods that can achieve that goal, such as Minimum-cut method, Hierarchical clustering, Girvan-Newman algorithm, etc. 
Minimum-cut method is one of the oldest algorithms for dividing networks into parts. This method uses in load balancing for parallel computing in order to minimize communication between processor nodes. However, this method always finds communities regardless of whether they are implicit in the structure, and it can only find a fixed number of them. So it is less than ideal for finding community structure in general networks [4].

Hierarchical clustering is another method for finding community structures in network. This method detects the community by defining a similarity measure quantifying some (usually topological) type of similarity between node pairs.



Figure 1 An example of nodes relations. Small circles represent nodes. Short lines represent the relation among them.

The Girvan-Newman algorithm is one commonly used algorithm for finding communities [12]. It identifies edges in one network that lie between communities and then removes them, leaving behind just the communities themselves. But this method runs slowly which makes it impractical for networks of more than a few thousand nodes [11].

Modularity maximization is one of the most widely used methods for community detection [11]. Modularity is a benefit function that measures the quality of a particular division of a network into communities. This method detects the community structure of high modularity value by exhaustively searching over all possible divisions [8].

In this paper we adopt modularity maximization as the social group detection method. Modularity maximization is one of the most widely used methods for group detection [11].

Modularity is a benefit function that measures the quality of a particular division of a network into groups. This method detects the group structure of high modularity value by exhaustively searching over all possible divisions [8]. 
In real networks, the modularity value is usually in the range [0.3, 0.7]; 1 means a very strong group structure and 0 means no better than random.

\section{MOBILITY MODEL SETUP}

\subsection{Mobility Scenario Description}

Usually peer nodes in one group act in concert, for example, these nodes may go from office to restaurant together for lunch, maybe after lunch they will have a rest and then go from restaurant to cinema for entertainment.

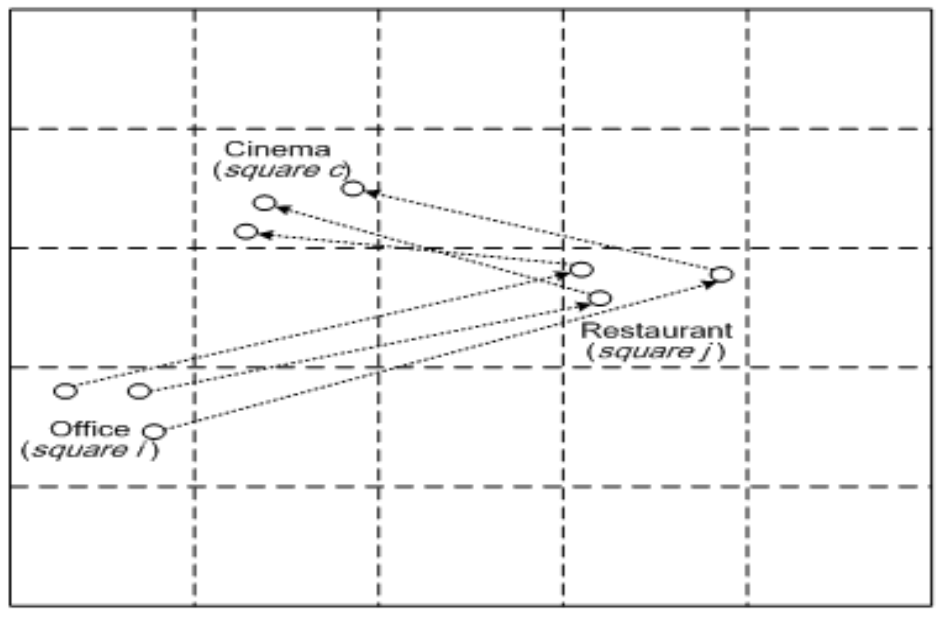

Figure 2 Illustration of mobility scenario

In terms of this realistic mobility scenario, this paper here models the office, restaurant and cinema to be small squares as shown in figure 2 in simulation scenario.

When the group moves from office (square $\mathrm{i}$ ) to restaurant(square $\mathrm{j}$ ), the restaurant (square $\mathrm{j}$ ) will be regarded as group-destination for all the peer nodes.

When the group continues to move from restaurant the cinema, one new square (square c) in the simulation will be chosen as the new group-destination corresponding to the cinema.

It should be noted that peer nodes in the same group also have their own self-destination. This means that these nodes self-destinations may be different positions corresponding to different points in the group-destination square.

In realistic scenario, another important phenomenon is as follows: when nodes move towards the destinations, some of them can move fast and some of them perhaps move slowly. This results that these faster nodes can reach their self-destinations earlier than the slower nodes. After arriving, these faster nodes would wait other slower nodes for a while before departure for next new group destination. 


\subsection{Mobility Model Abstraction}

Before moving, each group will randomly choose one small square in the simulation area to be its group-destination. And then each peer node in the group will choose one position in that small square to be its self-destination individually. Once they know their self-destinations, each node will move straightly towards its goal at some speed value. Because of the speed and destination position difference of nodes in one group, they can't ensure that all of them will reach their selfdestinations at the same time. So the former arriver will pause for a period of time to wait until all his peer partners arrive. Upon all the members of the group achieve arrival, they will choose a new small square to be their next common destination and move towards it.

\subsection{Mobility Mathematical Model}

Assume there are total $\mathrm{N}$ nodes in one group, the $n^{\text {th }}$ node is denoted as node $n(1 \leq n \leq N)$. The beginning square is denoted as $S_{j}$, so the beginning position of node $\mathrm{n}$ can be denoted as $P_{n}(t \mid t=0) \in S_{i}$. The group-destination square is denoted as $S_{j}$, the self-destination of node $n$ in $S_{j}$ is denoted as $P_{n}(t \mid t=0) \in S_{i}$.

At time $t(t \geq 0)$ the position of node $n$ is a function of coordinate $X_{d n}(t)$ and $Y_{d n}(t)$ which can be expressed as follows:

$$
P_{n}(t+1)=\left[\begin{array}{c}
X_{n}(t+1) \\
Y_{n}(t+1)
\end{array}\right]=\left[\begin{array}{c}
X_{n}(t)+V_{n}(t) \cdot \cos \Phi_{n}(t) \\
Y_{n}(t)+V_{n}(t) \cdot \sin \Phi_{n}(t)
\end{array}\right]
$$

Where

$t \quad$ is the current moment;

$t+1$ is the next moment;

$\Phi_{n}(t)$ is the direction function of node $\mathrm{n}$ which can be defined as

$$
\Phi_{n}(t)=\operatorname{arctg}\left(\frac{\left|Y_{d n}-Y_{n}(t)\right|}{\left|X_{d n}-X_{n}(t)\right|}\right)
$$

$V_{n}(t)$ is the velocity function which obeys normal distribution.

\subsection{Speed Choice}

The research in [13] has shown that the walking speed of a pedestrian obeys normal distribution. The measurement in [14] shows that the mean speed of a walking pedestrian is a range which is from $1.16 \mathrm{~m} / \mathrm{s}$ to $1.58 \mathrm{~m} / \mathrm{s}$ representing walking normally or walking fast.

In [15], the Manual of Uniform Traffic Control Devices (MUTCD) shows that pedestrians walk with a normal speed of $1.2 \mathrm{~m} / \mathrm{s}(4 \mathrm{ft} / \mathrm{sec})$. [10] indicates a statistics that walking speed for younger pedestrians (ages 14 to 64$)$ was $1.25 \mathrm{~m} / \mathrm{sec}(4.09 \mathrm{ft} / \mathrm{sec}$ ); for older pedestrians (ages 65 and over) it was $0.97 \mathrm{~m} / \mathrm{sec}(3.19 \mathrm{ft} / \mathrm{sec})$. For designing purposes values of $1.22 \mathrm{~m} / \mathrm{sec}(4 \mathrm{ft} / \mathrm{sec})$ for younger pedestrians and $0.91 \mathrm{~m} / \mathrm{sec}(3 \mathrm{ft} / \mathrm{sec})$ for older pedestrians are appropriate[10]. 
In this paper, the mean value of walking speed of the node $n, \mu_{n_{v}}$, equals to $1.22 \mathrm{~m} / \mathrm{s}$ [10] and its standard deviation $\sigma_{n_{v}}$ is uniformly chosen in the range of $\left[\begin{array}{ll}0 & 0.26 \mathrm{~m} / \mathrm{s}\end{array}\right]$ [13]. So the speed of node $n$ at time $\mathrm{t}$ is

$$
V_{n}(t) \sim N\left(\mu_{n_{v}}, \sigma_{n_{v}}\right)
$$

The probability density function of $V_{n}(t)$ can be expressed as:

$$
f(v)=\frac{\exp \left(-\frac{\left(v-\mu_{n_{v}}\right)^{2}}{2 \sigma_{n_{v}}^{2}}\right)}{\sigma_{n_{v}} \sqrt{2 \pi}}
$$

\section{MODEl CHARACTER ANALYSIS}

\subsection{Classical Individual Mobility Model-RWP}

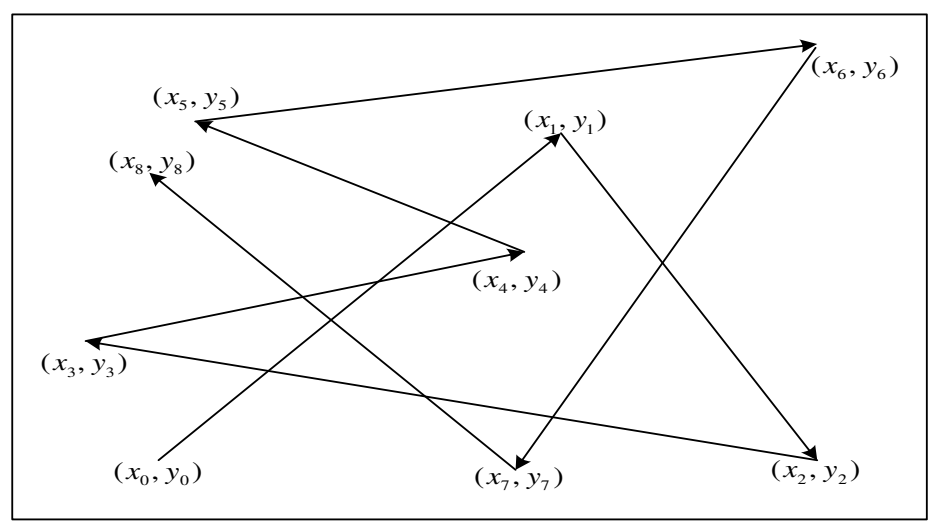

Figure 3 Illustration of RWP mobility

Josh et al. [8] present a Random Waypoint model. In this model a mobile node is initially placed at random location in the simulation area with a destination and speed according to the random distribution. Then the mobile node moves from its current location to it's destinations via a straight line. After arrival at the destination, the mobile node determines a pause time according to a random distribution, and after that chooses a new destination.

\subsection{Analysis and Comparison}

The proposed group model in this paper can be regarded as one improvement version of RWP. To analyze the character of these two mobility models, two important parameters inter-contact time and contact time are adopted. These two parameters describe the characteristics of connection opportunities of the network, i.e. how many and when do they occur, how often and how long. Contact time is defined as the time interval during which the two nodes can keep contact. The time interval from this contact to next one is defined as inter-contact time during which nodes can't communicate. [1] These two parameters are very important for opportunistic 
network. Contact time can help us determining the capacity of opportunistic networks. Intercontact time is a parameter which strongly affects the feasibility of the opportunistic networking.

The new mobility model in this paper is named as Peer Node Group Mobility Model (PNGM) here. We let each mobility model run 1000 seconds for one experiment and made 50 times similar experiments for each mobility model. Figure 4 and Figure 5 give the contact time distribution and inter-contact time distribution in different coordinate systems. In Fig. 4 (a), we can see that the new mobility model' inter-contact time distribution behave an exponential distribution plot using log-log coordinate system. In Fig. 4 (b), the contact time distributions show more evident difference. The RWP mobility models' curves are still exponential-like curves. In contrast, the shape of PNGM model' curves are changed. The PNGM model's curve is one kind of transition from exponential-like curve to power- law-like curve. This shows PNGM model behaves stronger group character than RWP model. In Fig. 5 we can see the difference between the curves more clearly using semi-log coordinate system. The exponential nature of the inter-contact time shown in Fig. 4 (a) becomes straight line form in Fig. 5 (a).

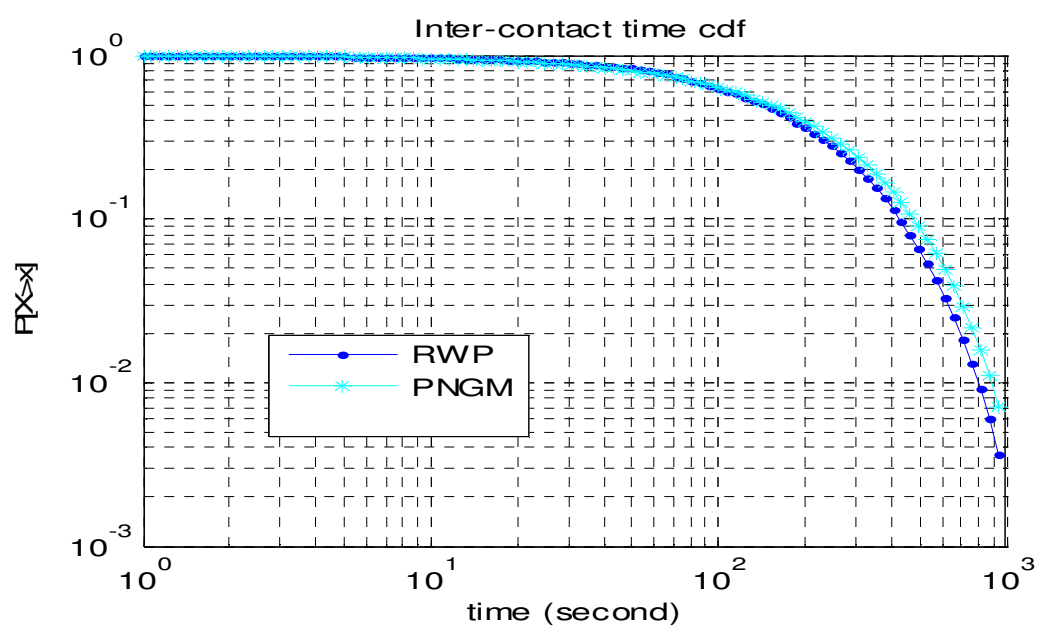

Fig 4 (a)

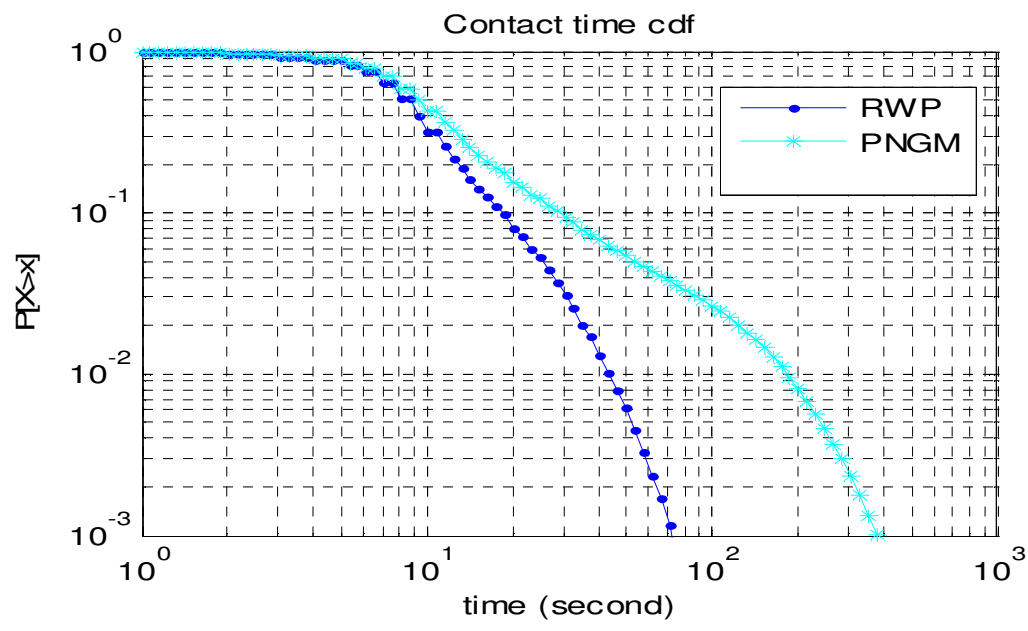

Fig4 (b)

Figure 4. Inter-contact time cdf and contact time cdf in log-log coordinates. 


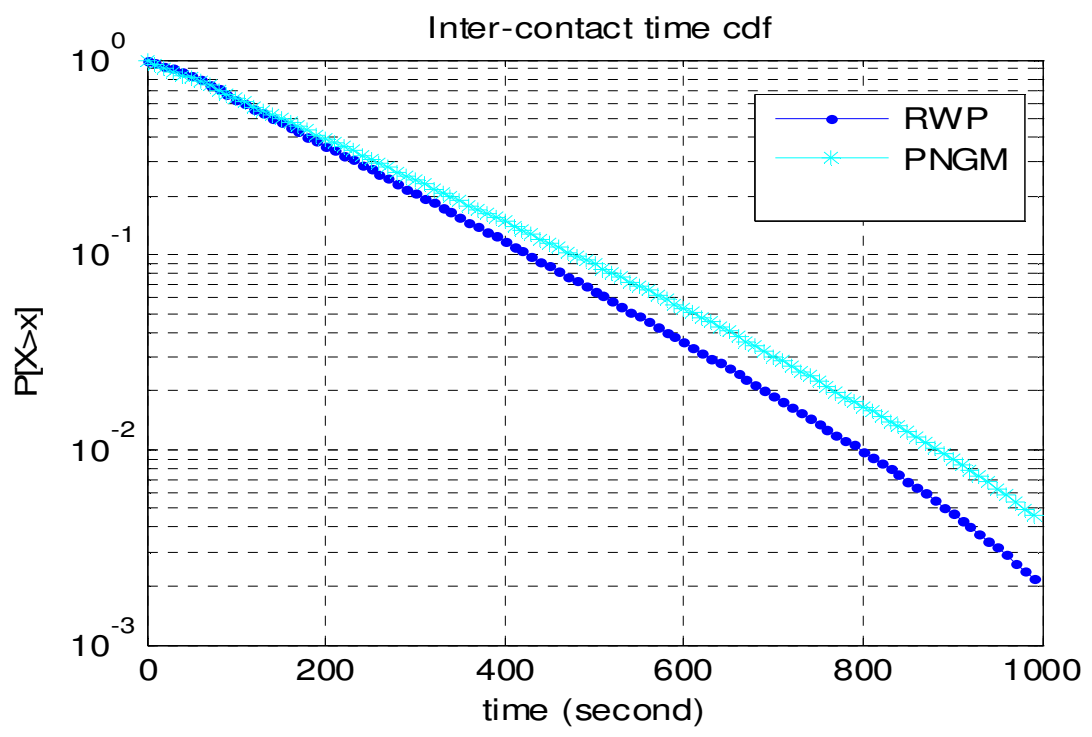

Fig 5 (a)

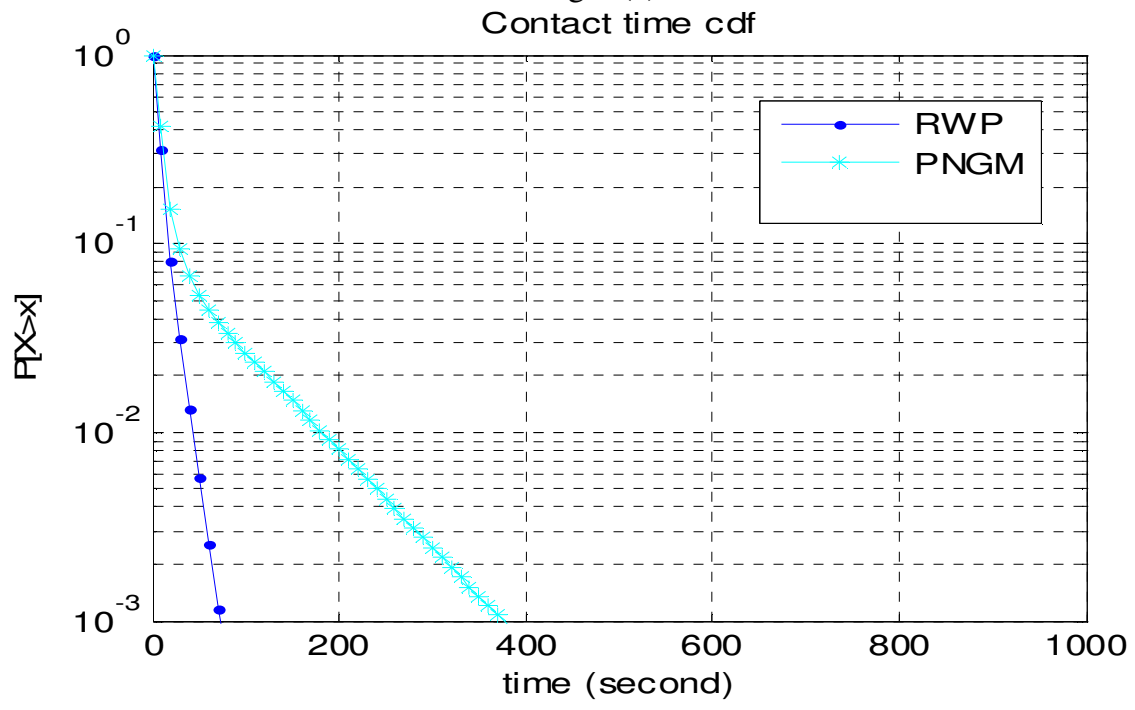

Fig5 (b)

Figure 5. Inter-contact time cdf and contact time cdf in semi-log coordinates.

\section{CONCLUSIONS}

In this paper, we proposed one new group mobility model with group character. We analyze its curves about contact time, inter-contact time. The curves show different shapes with the classical individual model RWP. By comparison, PNGM model shows obvious group character than RWP model.

\section{ACKNOWLEDGEMENTS}

This author would like to thank DFH satellite Co., Ltd. for the support to this paper. 


\section{REFERENCES}

[1] A. Chaintreau, P. Hui, J. Crowcroft, C. Diot, R. Gass, and J. Scott, "Pocket Switched Networks: Realworld mobility and its consequences for opportunistic forwarding," Technical Report UCAM-CLTR-617, University of Cambridge, Computer Laboratory, February 2005.

[2] J Broch, DA Maltz, DB Johnson, YC Hu, J Jetcheva," Multi-hop wireless ad hoc network routing protocols", in Proceedings of the ACM/IEEE International Conference on Mobile Computing and Networking (Mobicom 1998), pages 85-97, 1998.

[3] Y. Ko and N.H. Vaidya, "Location-aided routing (LAR) in mobile ad hoc networks," in Proceedings of the ACM/IEEE International Conference on Mobile Computing and Networking (Mobicom), pages 66-75, 1998.

[4] M. E. J. Newman, Detecting community structure in networks. Eur. Phys. J. B, 38, 321-330 (2004)..

[5] E. de Silva and M. Stumpf, "Complex networks and simple models in Biology", J. R. Soc. Interface, 2 (2005), pp. 419-430..

[6] N. Przulj, D. G. Corneil, and I. Jurisica, "Modeling interactome:Scale- free or geometric?", Bioinformatics, 20 (2004), pp. 3508-3515..

[7] D. J. Watts and S. H. Strogatz, "Collective dynamics of 'small-world' networks", Nature, 393 (1998), pp. 440-442.

[8] M.E.J. Newman and M. Girvan. "Finding and evaluaing community structure in networks". Physical Review E,68,2003.

[9] Mirco Musolesi, Cecilia Mascolo, "A community based mobility model for ad hoc network research", in Proceedings of the 2nd international Workshop on Multi-Hop Ad Hoc Networks: From theory To Reality (REALMAN '06), Florence, Italy, May 2006, pp 31-38.

[10] R.L. Knoblauch, M.T. Pietrucha, M. Nitzburg. Field studies of pedestrian walking speed and start-up time. Transportation Research Board Records No. 1538, 1996.

[11] Newman, M. E. J. Fast algorithm for detecting community structure in networks. Phys. Rev. E 69,066133 (2004)

[12] Girvan, M. and Newman, M. E. J., Community structure in social and biological networks, Proc. Natl. Acad.Sci. USA 99, 8271-8276 (2002).

[13] L. Henderson. The statistics of crowd fluids. Nature, Vol. no. 229, 381-383, 1971.

[14] Finnis, K.K. and Walton, D. Field observations of factors influencing walking speeds. Ergonomics, 2006.

[15] LaPlante, John and Kaeser, Thomas P. "A History of Pedestrian Signal Walking Speed Assumptions", in Proceedings of the 3rd Urban Street Symposium: Uptown, Downtown, or Small Town: Designing Urban Streets That Work, 2007.

\section{AUTHORS}

GuoDong KANG is an telecommunication engineer of DFH Satellite Co., Ltd., 100094, Beijing, China.

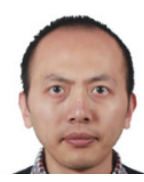

Guoliang KANG is an PhD student of University of Technology, Sydney 15 Broadway, Ultimo NSW 2007 